\title{
Exploiting Orientational Redundancy in Multiview Video Compression
}

\author{
Chi Wa Leong, Behnoosh Hariri*, Shervin Shirmohammadi \\ Distributed and Collaborative Virtual Environment Research Laboratory (DISCOVER Lab), University of \\ Ottawa, Ottawa, Canada. \\ * Corresponding author. Email: behnoosh@google.com \\ Manuscript submitted August 24, 2014; accepted February 13, 2015. \\ doi: 10.17706/ijcee.2015.v7.873
}

\begin{abstract}
This article introduces an approach for the acquisition and coding of multiview video. Multiview video systems consist of several cameras simultaneously capturing a single scene. Therefore a significant level of inter-view redundancy is present among the videos that can be exploited into video compression. Inspired by the idea of motion estimation in MPEG4 video compression, we introduce the idea of rotation estimation and compensation that is used in conjunction with motion estimation and compensation in order to remove spacial as well as temporal redundancies from the compressed video. The main question to be answered is how to choose the best sequence of compression among the frames when both time and space domains are involved. In this article, we model the above problem as a minimum cost graph traversal problem where cameras are considered as graph nodes and the cost of an edge connecting two cameras is inversely proportional to the similarity between the videos recorded by those cameras. We will then find the solution of this problem as the optimal traversal sequence that result in a high compression ratio.
\end{abstract}

Key words: Multiview video, 3D video, orientational redundancy.

\section{Introduction}

Multiview video applications have recently gained significant popularity in both academia and the industry. Experts believe that the future of video is 3D/multiview, a fact that has been clear from both academia and industry proponents of video [1]. The phenomenal success of the recently-released movie Avatar, which quickly became the best-selling movie in the history of cinema, has both demonstrated and fueled interest in multiview video. Other 3D movies have followed the suit, indicating 3D video has huge potential for the short and long term future. Many companies already offer 3D televisions and 3D Blu-ray players. In addition to movies, multiview video also has applications in virtual reality, tele-conferencing, and tele-immersive systems, adding a level of realism in human-to-human interaction possible through these applications. Such applications are required to integrate various technologies such as multiview video acquisition, coding, compression, transmission and rendering. Video recordings captured at a source are pre-processed before being transmitted. Such processing includes image processing (background subtraction, rectification, etc.), signal filtering recognition of user inputs for navigational purposes, synchronization of different media, and content coding. The processed data are then transmitted over the network to the other side where it will be decoded and rendered using appropriate output devices.

Multiview video consists of several videos of an object that have been simultaneously recorded using several cameras. Therefore, a high level of redundancy exists among these videos that can be exploited for 
video compression. We introduce the concept of rotation estimation based on the fact that the videos recorded by different cameras capture the same object from different rotational angles. In other words, each camera records a 2D video that is the mapping of a 3D scene onto a different plane. Therefore, each 3D point is mapped onto a camera 2D plane that is specified by the position and orientation of the camera. The concept of rotation vector can then be used in conjunction with the motion vector in order to remove both temporal and spacial redundancies from the compressed video. We start by looking at existing approaches.

\section{Related Work}

The growing interest in 3D television (3DTV) and free viewpoint video (FVV) has driven up the demand of multiview video (MVV) [2]. Multiview video coding (MVC), an effort of the Joint Video Team (JVT) with members originating from both the ITU-T Video Coding Experts Group and ISO/IEC MPEG, is the standard that governs the encoding/decoding, transmission and storage of such type of video data. The main objective of MVC is to specify the requirements for the standard, and provide guidelines on the design of the required codec.Kubota et al. [3] have provided an overview of the MVC standard and a survey of research efforts. The authors characterize multiview video as a bulky media which, with a significant amount of redundant data, makes compression both a possibility and a necessity. Magnor et al. [4] propose a new multiview image coding approach that is based on the knowledge of 3D scene geometry. Florencio et al. [5] use an estimate of the viewer position to compute the expected contribution of each pixel to the synthesized view, and encode the macroblocks of each camera views with the resolution proportional to the likelihood that the macroblock is used in the synthetic image. Luo et al. [6] adopt a wavelet transform and rebinning approach to compress concentric mosaics. Maitre et al. [7] introduce a wavelet-based joint estimation and encoding approach for texture image and depth maps, while Li et al. [8] propose a video signal compression based on a new variation of the 3-Dimensional Discrete Cosine Transform (3D-DCT).

The search for an optimal encoding order has also been going on in parallel. Bai et al. [9] propose a neighbor-based search algorithm which produces an optimal encoding order. The search algorithm considers all possible sequences and selects the one which is highly correlated to its neighbors. The process continues until all the sequences are selected. Li et al. [10] consider modeling the encoding sequence problem as traversing a directed graph connecting the recorded images. A node traversal starting from the root node produces the sequencing of the views. For the spatial/view dimension, I and P frames are used while for the temporal dimension, hierarchical B frames are specified. Kang et al. [11] consider the image frames from all the views in a Group of Pictures (GOP). An original graph is then constructed to relate all these images. Prim's algorithm is used to extract the minimal spanning tree for the GOP.

Our work is different from the previous works in that we introduce the concept of optimized compression based on rotation estimation at the encoder, and rotation compensation at the decoder. To the best of our knowledge, no other work has utilized rotational redundancy of information between multiple views in order to achieve higher compression of multiview video. In the next section, we present the design behind our proposed rotation estimation approach.

\section{Proposed Compression Scheme}

Assume that point $P$ is a physical point on the object being recorded, and that $P$ with $3 \mathrm{D}$ coordinates $\left[P_{x} P_{y} P_{z}\right]^{T}$ is mapped to a 2D image point $P_{i}$ in the video recorded by the $i^{t h}$ camera. Camera $i$ is characterized by a $3 \times 4$ perspective projection matrix $\Pi_{i}=\left[Q_{i} \mid q_{i}\right]$ where $Q_{i}$ is a $3 \times 3$ matrix describing the rotational orientation of the camera, and $q_{i}$ is a $3 \times 1$ vector describing the location of the camera's optical center [12]. The camera projection matrix determines the transformation from $P$ to the new point in the recorded 2D pictures. For two cameras $i$ and $j$, such relation has been described in (1) and (2): 


$$
\begin{gathered}
p_{i}=\prod_{i} P \\
P_{j}=\prod_{j} P
\end{gathered}
$$

The relationship between the mapped locations $P_{i}$ and $P_{j}$ can be approximated by an affine transformation as described in (3):

$$
\left[\begin{array}{l}
x_{j} \\
y_{j}
\end{array}\right]=\left[\begin{array}{ll}
a_{00} & a_{01} \\
a_{10} & a_{11}
\end{array}\right] \cdot\left[\begin{array}{l}
x_{i} \\
y_{i}
\end{array}\right]+\left[\begin{array}{l}
b_{0} \\
b_{1}
\end{array}\right]=\left[\begin{array}{lll}
a_{00} & a_{01} & b_{0} \\
a_{10} & a_{11} & b_{1}
\end{array}\right] \cdot\left[\begin{array}{c}
x_{i} \\
y_{i} \\
1
\end{array}\right]
$$

The matrix $A=[a \mid b]$ can be estimated if we identify enough feature points in both images. Assuming that we deal with $N_{\text {feat }}$ number of feature points in both images, we can describe the following transformations among the two images:

$$
\begin{aligned}
{\left[\begin{array}{cccc}
x_{j, 0} & x_{j, 1} & \cdots & x_{j, N_{\text {feat }}-1} \\
y_{j, 0} & y_{j, 1} & \cdots & y_{j, N_{\text {feat }}-1}
\end{array}\right] } & =A \cdot\left[\begin{array}{cccc}
x_{i, 0} & x_{i, 1} & \cdots & x_{i, N_{\text {feat }}-1} \\
y_{i, 0} & y_{i, 1} & \cdots & y_{i, N_{\text {feat }}-1} \\
1 & 1 & \cdots & 1
\end{array}\right] \\
X_{j}=A X_{i} \Rightarrow A & =X_{j} X_{i}^{T}\left(X_{i} X_{i}^{T}\right)^{-1}
\end{aligned}
$$

Even though $X_{i}$ is not a square matrix, $A$ can be determined by finding the pseudo-inverse $\left(X_{i} X_{i} T\right)^{-1}$. Once $A$ is found, the inverse mapping from $P_{j}$ to $P_{i}$ can also be calculated as follows:

$$
\left[X_{i}\right]=\left[a^{-1} \mid-a^{-1} b\right] \cdot\left[\begin{array}{c}
X_{j} \\
1
\end{array}\right]
$$

In situations where the camera positions are fixed, transformation A depends on the variation of the video content. Its values can be recalculated whenever there is a significant variation. It should be noted that due to the limited visibility range of each camera, all points in the 3D image mapping to camera $i$ are not present in the recorded image; i.e., camera i only captures a part of this projection. Therefore, as already known intuitively, there is not a hundred percent redundancy in the images recorded by different cameras. The correlation among the recorded videos between two cameras is inversely proportional to the distance and rotation angle of those cameras. The basic idea behind our compression scheme is to rotate the video frames recorded by different cameras in order to align them to a selected reference video frame. The difference with respect to the reference frame is considered as the compressed frame for that view. It should be noted that the selection of a proper sequencing for compression of videos is also a factor that has a significant impact on the compression efficiency. Our proposed scheme aims at finding the best compression sequence among the temporal and spacial frames. The best compression sequence in our case is defined as the sequence in which neighboring frames are chosen to be as similar as possible. To explain this, we will use the Ballroom multiview sequence which is captured simultaneously using 8 cameras, the first 4 frames of which are shown in Fig. 1. We will use this as an example to show how we achieve higher compression with rotation estimation, which is done in two parts: 1- calculation of the similarity measure among the views, and 2- selection of the compression sequence.

\subsection{Similarity Measure}

In order to estimate the best encoding order, a similarity measure should first be defined between each pair of images. The similarity measure should reveal the amount of common information between two 
frames irrespective of any possible differences that can be compensated by pre-processing such as transformation or lighting compensation. Therefore, we will apply both spatial and temporal pre-processing steps prior to similarity measurements. The required pre-processing in the space domain is referred to as rectification that is used to align two images that have been recorded from different viewpoints. This step is generally referred to as rotation estimation and compensation.

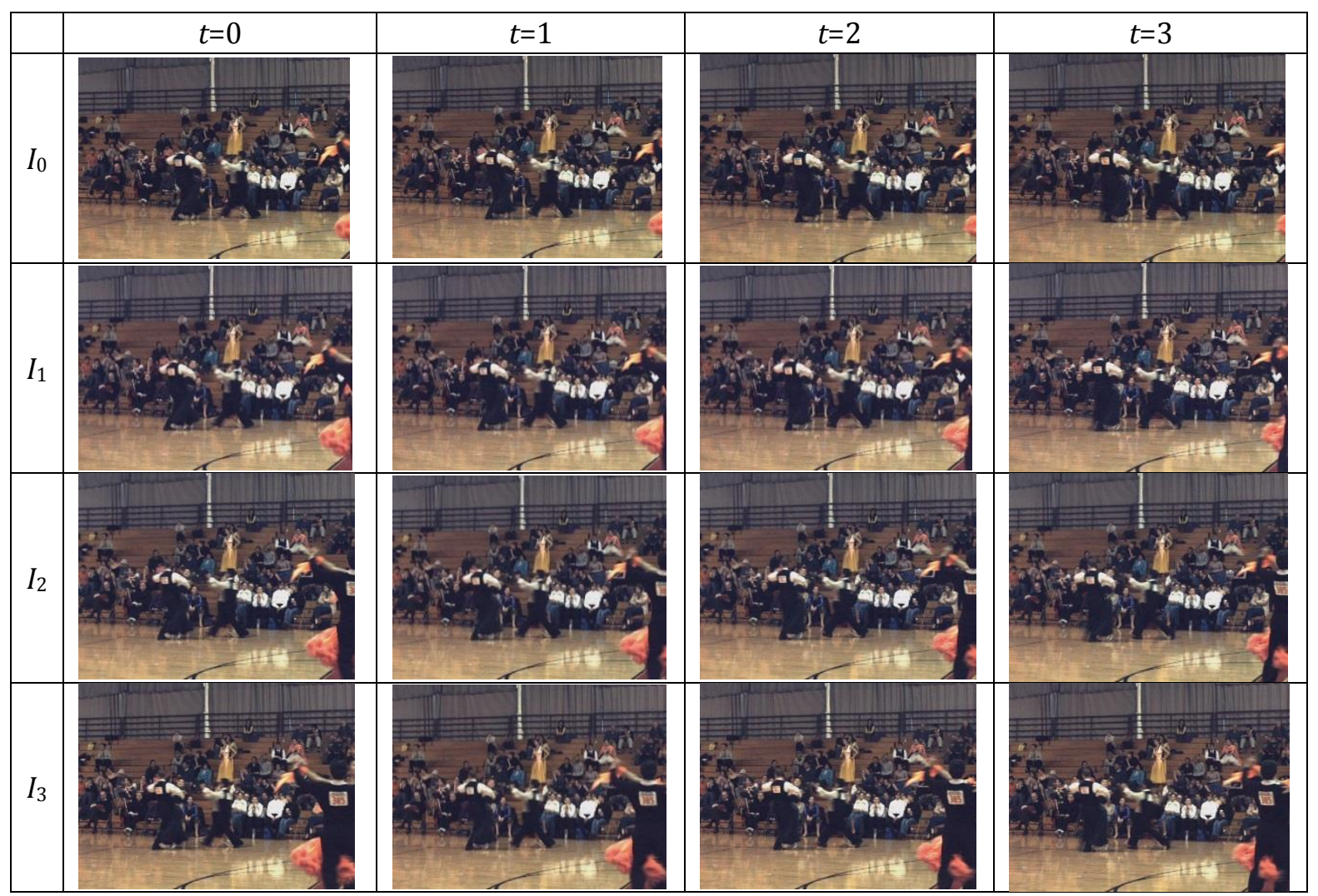

Fig. 1. The first four frames ( $t=0$ to 3 ) of the Ballroom sequences captured by Cameras 0 to 3 .

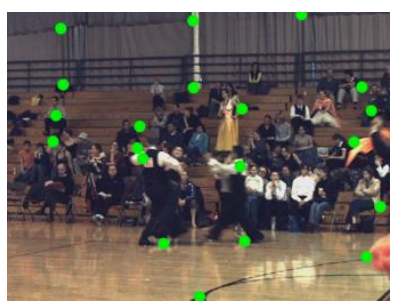

(a)

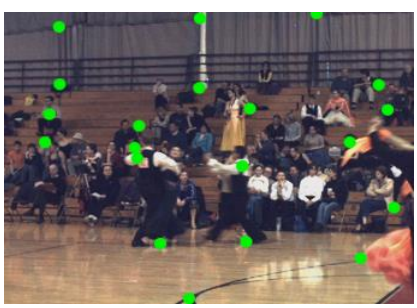

(b)

Fig. 2. Feature points manually located in two synchronized images from (a) camera0 (b) camera1.

Here we provide an example of rectification between the frames demonstrated in Fig. 2. Twenty feature points have been located on images $I_{0}$ and $I_{1}$ at $t=0$ as can be seen in the figure. Let $I_{0}$ be the reference frame for $I_{1}$. The affine transformation that can be used to warp $I_{0}$ towards $I_{1}$ is defined by matrix $A$ :

$$
A_{1 \leftarrow 0,0}=\left[\begin{array}{ccc}
1.013016 & 0.061877 & -1.872520 \\
0.000433 & 0.998908 & 2.736671
\end{array}\right]
$$

where $A_{x \leftarrow y, t}$ represents $A$ 's transformation from Camera $y$ to camera $x$ at time $t$. Since the physical configuration of the camera array is in a linear 1D arrangement, the rotational component, $a$, of the transformation as described in (5) is very close to an identity matrix, and the transformation between the 
two cameras' frames can mainly be described by the translation vector $b$. The original $I_{0}$ and $I_{1}$ together with the warped $I_{0}$ are shown in Fig. 3.

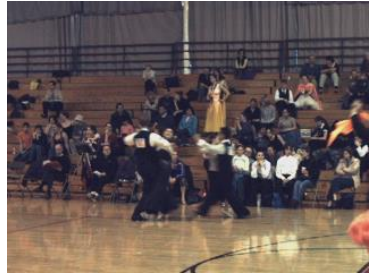

(a)

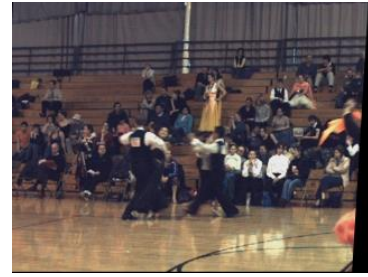

(b)

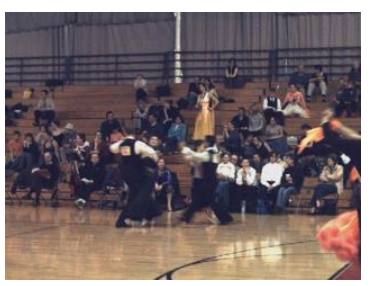

(c)

Fig. 3. (a) Original left image $I_{0}$, (b) I0 warped towards $I_{1}$, (c) Original right image $I_{1}$.

Bilinear interpolation is used when the warping function does not produce integer coordinates. There is a slight decrease in the difference measure when the warped $I_{0}$ is used as a reference. This characteristic can be further explored when calculating the similarity of the two images.

Following rectification, we will use motion compensated sum of squared differences (MCSSD) [11] in order to measure the difference between the frames after temporal motion compensation. Consider two equal-sized images, $I_{i}$ and $I_{j}$. If both images are divided into NB non-overlapped sub-blocks, the block-wise $\operatorname{MCSSD}_{k}$ for the $k^{\text {th }}$ sub-block can be defined as:

$$
\operatorname{MCSSD}_{k}\left(I_{i}, I_{j}\right)=\sum_{(x, y) \in B L O C K_{k}}\left[I_{i}(x, y)-I_{j}\left(x+x_{k}, y+y_{k}\right)\right]^{2}
$$

where $\left(x_{k}, y_{k}\right)$ is the 2D motion vector for the $k^{\text {th }}$ sub-block. This is the vector that minimizes the block-wise $\operatorname{MCSSD}_{k}$ over a search window WIN $k$ :

$$
\left(x_{k}, y_{k}\right)=\arg \min _{\left(x_{k}, y_{k}\right) \in W I N_{k}} \operatorname{MCSSD} D_{k}\left(I_{i}, I_{j}\right)
$$

The overall MCSSD for the entire image is therefore defined as:

$$
\operatorname{MCSSD}\left(I_{i}, I_{j}\right)=\sum_{0 \leq k \leq N_{B}-1} \operatorname{MCSSD} D_{k}\left(I_{i}, I_{j}\right)
$$

This is a positive number reflecting proportionately how different the two images $I_{i}$ and $I_{j}$ are, and can be used as a distance measure. Its value is zero when the two images are completely identical. Since MCSSD is not symmetric, we can further define a symmetric distance measure which takes the average of the two possible MCSSD values:

$$
\operatorname{dist}(i, j)=\operatorname{dist}(j, i)=\frac{\operatorname{MCSSD}\left(I_{i}, I_{j}\right)+M \operatorname{CSSD}\left(I_{j}, I_{i}\right)}{2}
$$

To obtain an approximate range for the value of MCSSD, consider the situation where we would like to measure the distance between two color images, both of which are of size $640 \times 480$ in 3 channels (RGB). Assume that a single byte is dedicated to represent each channel, whose intensity value ranges from 0 to 255 . Also, assume the two images are different, and on the average there is a difference of $2 \%(255 \times 2 \% \approx 5)$ in the intensity value when compared pixel by pixel. This amounts to an approximate total MCSSD of $640 \times 480 \times 3 \times 52 \approx 2 \times 10^{7}$. This quantity indicates that the MCSSD will be in the order of $10^{7}$ for a color image of a similar size. This is indeed the range of values we obtain in the experimental section, and therefore we 
verify the validity of our measured MCSSD values. The distance measure may be larger or smaller, depending on the actual difference that exists between the two images.

The MCSSD between $\left(I_{1}, I_{0}\right)$ in Fig. 2 is 15.01 , while $\operatorname{MCSSD}\left(I_{1}, W_{1-0}\left(I_{0}\right)\right)$ is 13.83 , where $W_{1-0}(\bullet)$ denotes the warping function from the view point of Camera 0 to that of Camera 1 . Therefore, we can see that MCSSD decreases after the warping operation as a result of revealing the hidden similarities.

Instead of manually specifying feature points on the images, an alternative is to use Scale Invariant Feature Transform (SIFT) descriptors [13]. The detected SIFT features of $I_{2}$ and $I_{3}$ at $t=47$ are shown in Fig. 4(a) and (b), respectively. The correspondence between the feature points of the two images can be obtained using a search procedure based on kd-tree. The matching of 146 feature points is shown in Fig. $4(c)$. The coordinates of these corresponding feature points can then be substituted into both sides of (4) to solve the transformation matrix $A$. In this case, $A$ is found to be:

$$
A_{3 \leftarrow 2,47}=\left[\begin{array}{lll}
1.020522 & 0.044407 & -5.601340 \\
0.001055 & 1.006317 & -2.324421
\end{array}\right]
$$

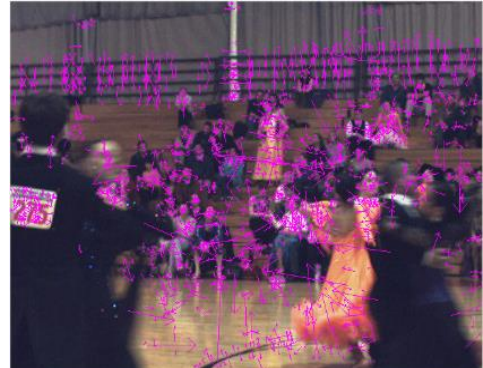

(a)

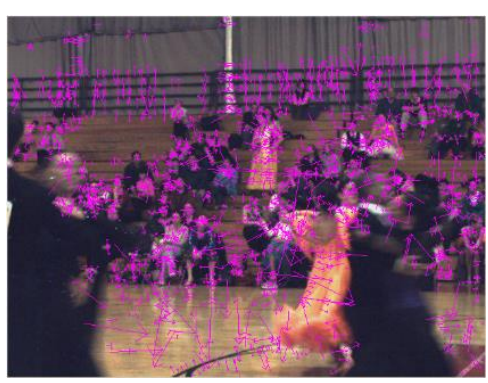

(b)

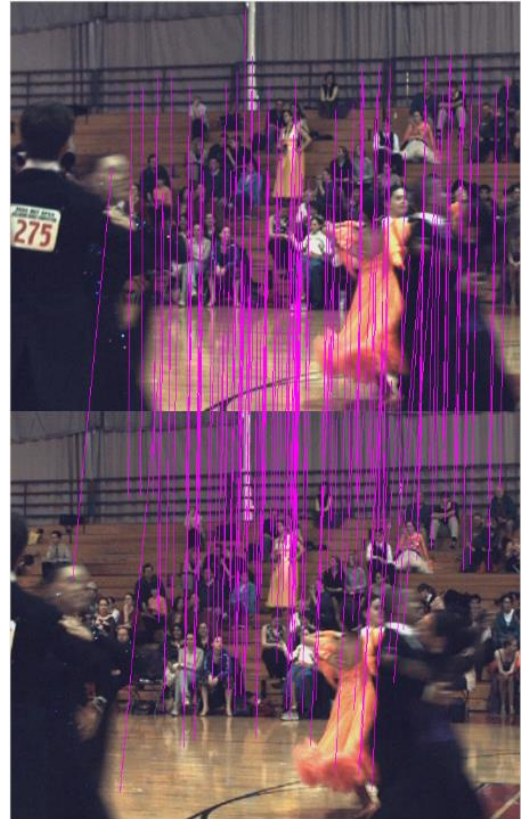

(c)

Fig. 4. (a) SIFT features of $I_{2}$; (b) SIFT features of $I_{3}$; (c) matching features.

Once again, we can observe that $a$ is very close to an identity matrix, as all cameras are in a 1D linear arrangement. In addition, $A_{3-2,47}$ is slightly different than $A_{1-0,0}$ because the video contents are different.

\subsection{Finding the Proper Compression Sequence}

After measuring the similarity between the videos, the next step is to define the best sequence of compression that achieves the highest compression rate (least amount of redundancy). In order to find the best encoding order, we model the problem as a graph traversal problem where each camera is a node and the weight of the edges connecting two nodes is defined to be the distance of the frames that they record:

$$
w_{i j}=\operatorname{dist}(i, j)=\operatorname{dist}(j, i)=w_{j i}
$$

where $w_{i j}$ refers to the weight between camera $i$ and $j$. The best camera traversal order maximizing the 
overall compression rate is the one that minimizes the overall distance traversed. The graph demonstrating the cameras and their initial connections is illustrated in Fig. 5 for the case of four cameras.

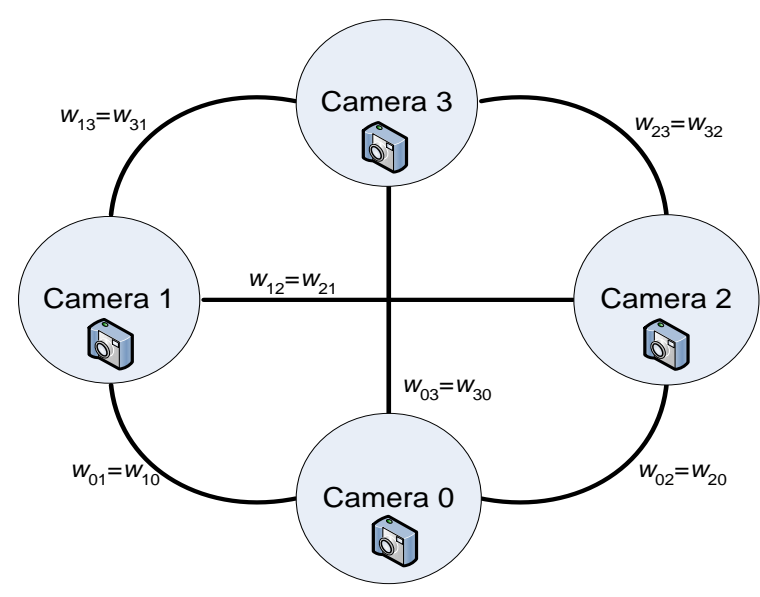

Fig. 5. The graph model of the sequencing problem.

To obtain the minimum cost traversal path on the tree, we calculate a minimum spanning tree (MST) of the graph and then perform a depth-first traversal on the tree. Consider the images at time instant $t$ to be $I_{i, t}$ for where $i$ represents the view number $\left(0 \leq i \leq N_{\mathrm{v}}-1\right)$ and $N_{v}$ is the number of views.

We first look at the encoding order at a fixed time instant; thus we can omit the time index in this discussion. The details of the procedure can be described in the following steps:

1) Defining the weights: Calculate $w_{i j}\left(0 \leq i, j \leq N_{v}-1\right)$ for all edges in the fully connected graph of Fig. 5.

2) Finding MST: Find MST of the graph using Prim's algorithm.

3) Selecting an appropriate root node: choose the node with the highest degree belonging to MST. If there is a tie, then choose the one with the total smallest outgoing weights.

4) Rearranging the MST: Put the chosen root node at the top of the tree and rearrange other nodes underneath according to the connectivity of the MST.

5) Finding the depth: Calculate the depth of all nodes in the tree starting from the leaf nodes, recursively from the leaf nodes with the depth of a leaf node $=0$, and the depth of node $i$ being:

$$
i=1+\max _{j \in\{\text { children of } i\}}[\text { depth of } j]
$$

6) Obtaining an encoding order: Perform a pre-order traversal of the tree starting from the root node. For the child nodes, traverse them in the order of ascending node depth (i.e. traverse shallower nodes first). This will have the advantage of minimizing the required encoder memory.The encoding order can be adapted to the time varying natures of the videos by having this procedure executed once every $N_{\text {period }}$ frames. The value of $N_{\text {period }}$ can be determined according to average rate of scenery changes in the videos.

\subsection{Specification of Frame Types}

As a potential point of random access to a video sequence, the first image of a GOP is usually specified as an I frame for the main reference view or a P frame for the dependent views. Within a GOP, in the temporal dimension hierarchy, bi-directional/bi-predictive B frames can be specified. The advantage of using a hierarchical structure is that the transmission of the layered information can be conveniently made adaptive to diverse network conditions and heterogeneous communication devices. As can be seen from Fig. 
7, each frame in the sequence is assigned a label and a layer number. Frames with a label of capital letter (I, $\mathrm{P}, \mathrm{B})$ are those which will be used as reference frames during encoding/decoding, while frames with a small letter label will not. The layer number for a frame can be used for synchronization: it can be specified that the encoding/decoding of all the frames in one layer be completed before processing of the next hierarchical layer is started. Merkle et al. [2] empirically show that immediate neighbors provide most of the correlation for motion and disparity compensations. As a result, we only consider frames that are in adjacent proximity to a frame. Given several candidate reference frames available in the reference list during the encoding of a macro-block $\mathrm{k}$, we choose the reference frame which minimizes the Lagrangian rate distortion cost as follows:

$$
\min _{F_{R} \in\{\text { Ref List }\}}\left[\operatorname{MCSSD}_{k}\left(F_{R}\right)+\lambda_{\text {motion }} \cdot R_{\text {motion }}\left(m_{k}\right)\right]
$$

where $F_{\mathrm{R}}$ is a reference image frame, $\lambda_{\text {motion }}$ is the Lagrangian parameter, and $R_{\text {motion }}$ is the number of bits required to transmit the motion vector $m_{k}=\left(x_{k}, y_{k}\right)$.

\section{Experimental Evaluation}

We implemented and used our proposed compression scheme on the four-camera scenario demonstrated in Fig. 6. The results are obtained using a combination of $\mathrm{C}++$, OpenCV, and JMVC. In the first scenario, no view transformation is performed between image frames of different views. The distance measure between the four images is shown in Table 1. The table is symmetric so we only show the upper triangular portion. The distance measure after the application of view transformation is shown in Table 2. It can be noted that the distance measure has decreased (ranging from 6\% to 38\%) when view transformation is introduced as demonstrated in Table 3.

Table 1. Distance Measure (No View Transformation)

\begin{tabular}{lllll}
\hline \hline Scale $\left(\times 10^{7}\right)$ & Image 0 & Image 1 & Image 2 & Image 3 \\
\hline Image 0 & & 13.71 & 31.51 & 42.32 \\
Image 1 & & & 14.49 & 28.29 \\
Image 2 & & & & 13.94 \\
\hline \hline
\end{tabular}

Table 2. Distance Measure (with View Transformation)

\begin{tabular}{lllll}
\hline \hline Scale $\left(\times 10^{7}\right)$ & Image 0 & Image 1 & Image 2 & Image 3 \\
\hline Image 0 & & 11.49 & 22.31 & 39.55 \\
Image 1 & & & 8.97 & 22.30 \\
Image 2 & & & & 9.81 \\
\hline \hline
\end{tabular}

Table 3. Distance Measure Decrease Using View Transformation

\begin{tabular}{lllll}
\hline \hline Scale $\left(\times 10^{7}\right)$ & Image 0 & Image 1 & Image 2 & Image 3 \\
\hline Image 0 & & $-16.19 \%$ & $-29.20 \%$ & $-6.55 \%$ \\
Image 1 & & & $-38.10 \%$ & $-21.17 \%$ \\
Image 2 & & & & $-29.62 \%$ \\
\hline \hline
\end{tabular}

The next step of the algorithm is constructing an MST for the undirected graph whose weights are initialized according to the entries of Table 2. The generated MST is shown in Fig. 6(a). Camera 2 has been selected as the root due to its smaller total outgoing weight. The tree is then rearranged and the depth for each node is calculated. The rearranged tree is shown in Fig. 6(b).The compression sequence is found to be 2-3-1-0. For a GOP of size 8, the relationship among the other frames for the four camera views is illustrated in Fig. 7(a). For this encoding structure, we have both the original views as well as the transformed views available during the encoding and decoding process. Whenever a spatial reference is needed, we can choose the desired version to use that is either the original view or both the original and the transformed views.To test the performance of our approach, we have used the MVC standard and two sequencing schemes: single anchor with camera view 0 chosen as the reference for all other views, and our proposed scheme. Both single anchor and our scheme achieve temporal plus spatial compression. We have also used the simulcast 
compression scheme with only temporal compression, all shown in Fig. 7. When applying our proposed sequencing scheme, we consider two types of generated compression sequences. We consider the case that the sequence has been obtained without view transformation (referred to as generated sequence (i), or GenSeq (i)), as well as the case with view transformation (referred to as generated sequence (ii), or GenSeq (ii)). The experimental parameters that are common to all encoders are shown in Table 4.

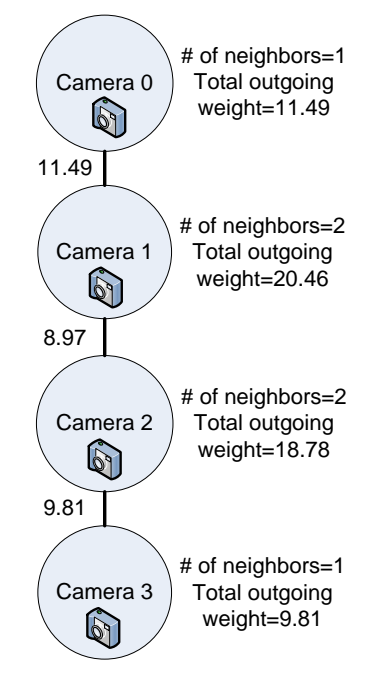

(a)

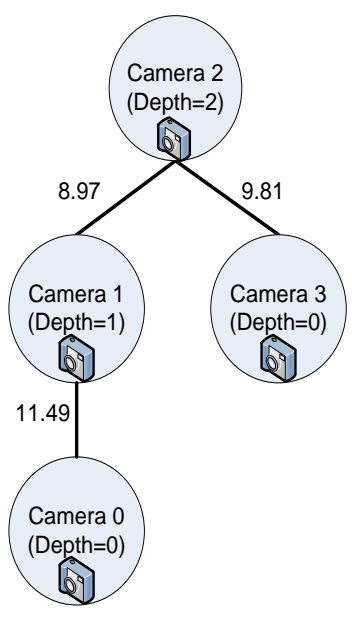

(b)

Fig. 6. (a) Original MST; (b) Rearranged MST.

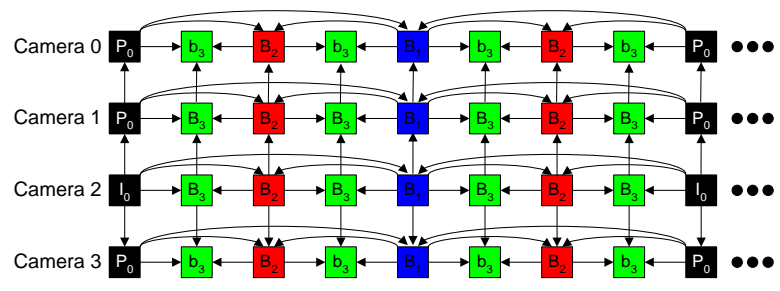

(a) Proposed sequencing scheme

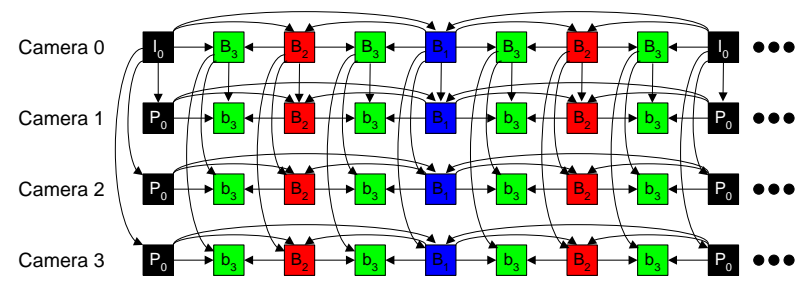

(b) Single anchor

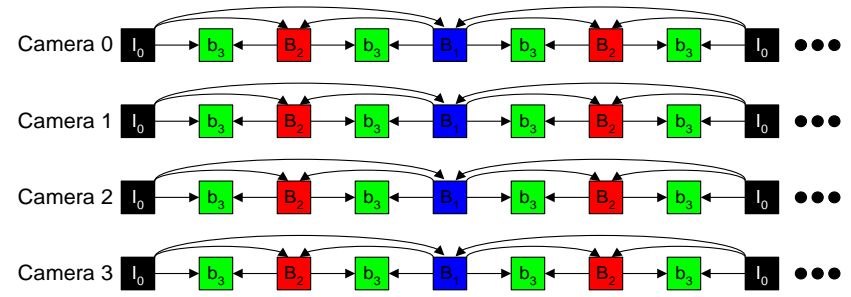

(c) Simulcast

Fig. 7. Structure of encoding sequence used in our experiment.

Table 4. Experimental Parameters

\begin{tabular}{lllll}
\hline \hline Sequence name & Ballroom & Image size & $640 \times 480$ & Sequence name \\
\hline Frame rate (fps) & 25 & GOP size & 8 & Frame rate (fps) \\
\# of cameras & $4 / 8$ & Camera array & 1D linear & \# of cameras \\
Search range & 32 & Intra-period & 64 & Search range \\
Basic QP & 29 to 35 & Frames & $4 \times 50=200$ & Basic QP \\
\hline \hline
\end{tabular}

Fig. 8 demonstrates the performance results and comparisons. As can be seen in the figure, our generated encoding sequences has an average gain of about $1.25 \mathrm{~dB}$ over the single anchor encoder, and about $1.50 \mathrm{~dB}$ over the simulcast encoder. Among our generated sequences, sequence (ii) has an additional $0.15 \mathrm{~dB}$ over 
sequence (i). A more detailed look at the data rate reduction is shown in Table 5 for a particular PSNR.

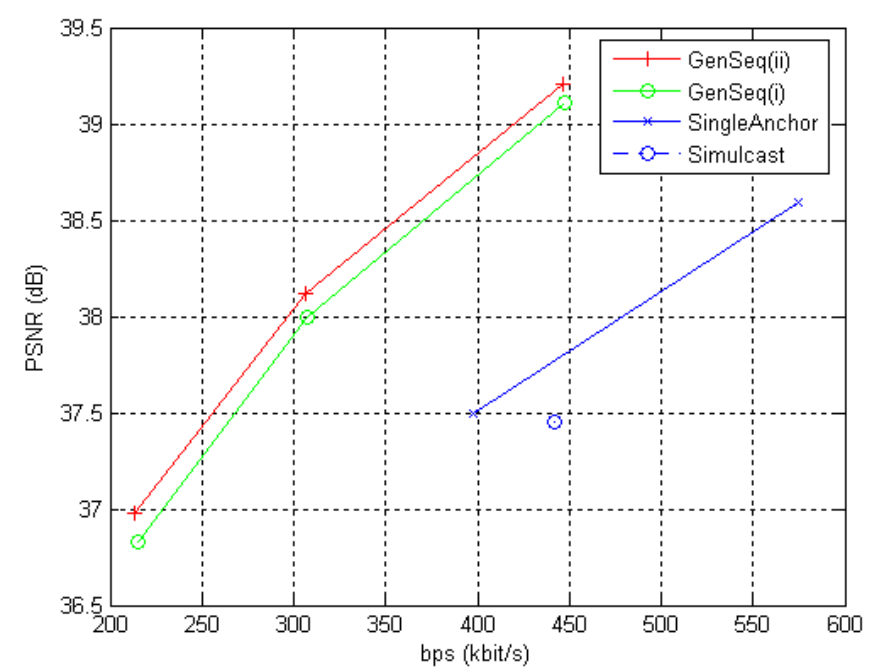

Fig. 8. Rate distortion performance of the encoding sequences.

Table 5. Data Rate Measured at Around PSNR $\approx 37.50 \mathrm{db}$

\begin{tabular}{lll}
\hline \hline Sequence & Avg. PSNR of YUV & Avg. bit rate/view \\
\hline Generated Sequence (ii) & $38.12 \mathrm{~dB}$ & $306.41 \mathrm{kbps}$ \\
Generated Sequence (i) & $37.99 \mathrm{~dB}$ & $307.56 \mathrm{kbps}$ \\
Single Anchor & $37.50 \mathrm{~dB}$ & $397.69 \mathrm{kbps}$ \\
Simulcast & $37.46 \mathrm{~dB}$ & $442.20 \mathrm{kbps}$ \\
\hline \hline
\end{tabular}

\section{Conclusions}

In this paper we have proposed a novel multi-view video compression scheme that achieves higher compression by removing the rotational inter-view redundancies among video streams simultaneously recorded from multiple views. The images captured from different views are first rectified using a transformation and then compressed in a specific order. This order is determined by an optimal stream encoding algorithm that is designed to enable the encoder to automatically decide on the ordering, and finds the best reference streams. Such optimal solution is found by modeling and solving the problem as a graph traversal problem among the camera views. Performance evaluations demonstrate the fact that the system achieves a better compression rate compared to simulcast and single anchor compression.

\section{References}

[1] Shirmohammadi, S., Hefeeda, M., Ooi, W. S., \& Grigoras, R. (2012). Introduction to special section on 3D mobile multimedia. ACM Transactions on Multimedia Computing, Communications, and Applications, $8(3 s)$.

[2] Merkle, P., Muller, K., Smolic, A., \& Wiegand, T. (2006). Efficient compression of multi-view video exploiting inter-view dependencies based on H.264/MPEG4-AVC. Proceedings of IEEE International Conf. on Multimedia and Expo (pp. 1717-1720). New York, USA: IEEE.

[3] Kubota, A., Smolic, A., Magnor, M., Tanimoto, M., Chen, T., \& Zhang, C. (2007). Multi-view imaging and 3DTV. IEEE Signal Processing Magazine, 24(6), 10-21.

[4] Magnor, M., Ramanathan, P., \& Girod, B. (2003). Multi-view coding for image-based rendering using 3-D scene geometry. IEEE Tran. Circuits and Systems for Video Technology, 13(11), 1092-1106.

[5] Florencio, D., \& Zhang, C. (2009). Multiview video compression and streaming based on predicted 
viewer position. Proceedings of IEEE International Conference on Acoustics, Speech and Signal Processing (pp. 657-660). New York, USA: IEEE.

[6] Luo, L., Wu, Y., Li, J., \& Zhang, Y.-Q. (2002). 3-D wavelet compression and progressive inverse wavelet synthesis rendering of concentric mosaic. IEEE Trans. on Image Processing, 11(7), 802-816.

[7] Maitre, M., Shinagawa, Y., \& Do, M. N. (2008). Wavelet-based joint estimation and encoding of depth-image-based representations for free viewpoint rendering. IEEE Trans. on Image Processing, 17(6), 946-957.

[8] Li, L., \& Hou, Z. (2007). Multiview video compression with 3D-DCT. Proceedings of ITI 5th Conf. on Information and Comm. Technology (pp. 59-61). New York, USA: IEEE.

[9] Bai, B., Boulanger, P., \& Harms, J. (2005). An efficient multiview video compression scheme. Proceedings of IEEE International Conference on Multimedia and Expo (pp. 836-839). New York, USA: IEEE.

[10] Li, D.-X., Zheng, W., Xie, X.-H., \& Zheng, M. (2007). Optimisinginter-view prediction structure for multiview video coding with minimum spanning tree. Electronics Letters, 43(23), 1269-1271.

[11] Kang, J.-W., Cho, S.-H., Hur, N. H., Kim, C. S., \& Lee, S. U. (2007). Graph theoretical optimization of prediction structure in multiview video coding. Proceedings of IEEE International Conference on Image Processing: Vol. VI (pp. 429-432). New York, USA: IEEE.

[12] Fusiello, A., Trucco, E., \& Verri, A. (2000). A compact algorithm for rectification of stereo pairs. Machine Vision and Applications, 12(1), 16-22.

[13] Hess, R. (2010). An open-source SIFT library. Proceedings of ACM Multimedia (pp. 1493-1496). New York, USA: ACM.

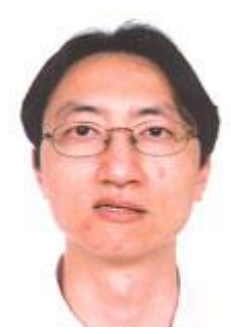

Chi Wa Leong was a PhD student at the Distributed and Collaborative Virtual Environment Research Laboratory (DISCOVER Lab), University of Ottawa, Canada, from 2009 to 2011. His research was focused on telepresence systems and networks, 3D/multiview video, and tele-immersive virtual environments.

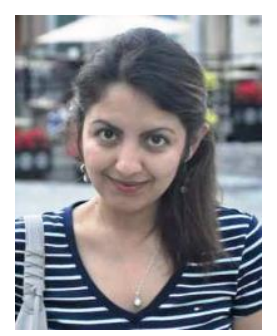

USA.

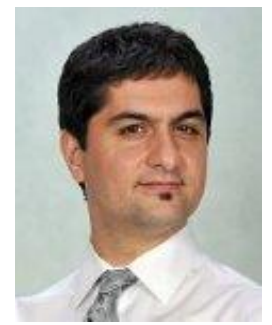

Behnoosh Hariri received her PhD degree in electrical engineering from Sharif University of Technology, Iran, in 2009, and was a MITACS elevate postdoctoral fellow at the Distributed and Collaborative Virtual Environment Research Laboratory (DISCOVER Lab), University of Ottawa, Canada, from 2009 to 2011. Her research was focused on gaming systems and networks, especially massively multiuser virtual environments, as well as vision based multimedia systems. She currently works at Google Inc. in New York,

Shervin Shirmohammadi received his $\mathrm{PhD}$ degree in electrical engineering from the University of Ottawa, Canada, where he is currently a full professor at the School of Electrical Engineering and Computer Science. He is the co-director of both the DISCOVER Lab, and the Multimedia Communications Research Laboratory (MCRLab), conducting research in multimedia systems and networking, specifically in gaming systems and virtual environments, video systems, and multimedia-assisted biomedical engineering. 
The results of his research have led to more than 250 publications, over 20 patents and technology transfers to the private sector, and a number of awards and prizes. He is the associate editor-in-chief of IEEE Instrumentation and Measurement Magazine, a senior associate editor of ACM Transactions on Multimedia Computing, Communications, and Applications, and an associate editor of IEEE Transactions on Instrumentation and Measurement. He had been an associate editor of Springer's Journal of Multimedia Tools and Applications from 2004 to 2012. He also has been chairs or serves on the program committee of a number of conferences in multimedia, virtual environments, and games. Dr. Shirmohammadi is a University of Ottawa gold medalist, a licensed professional engineer in Ontario, a senior member of IEEE, and a professional member of ACM. 